
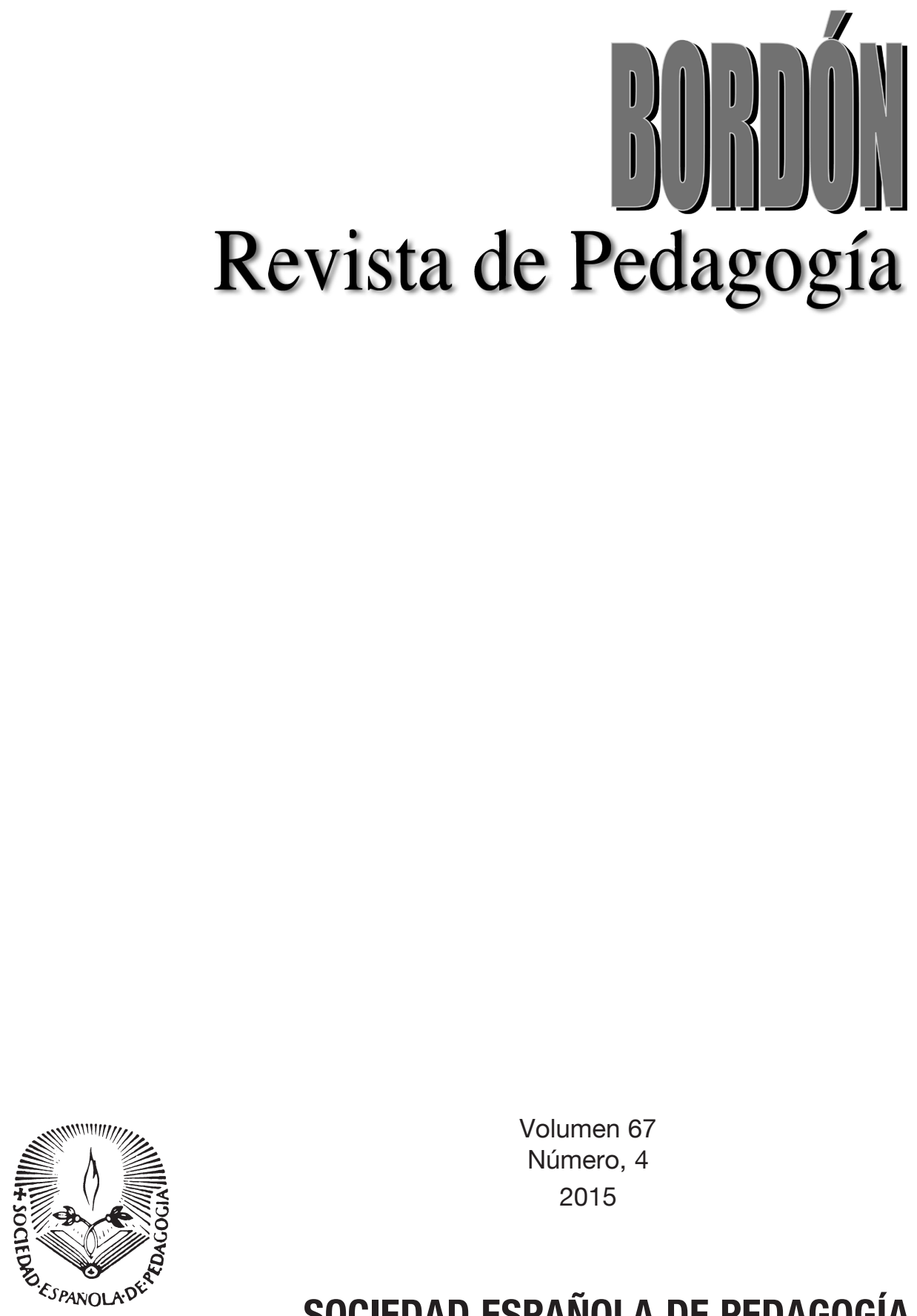

Volumen 67

Número, 4

2015

SOCIEDAD ESPAÑOLA DE PEDAGOGÍA 


\section{CAPACIDAD PREDICTIUA DEL AUTOCONCEPTO Y LA INTELIGENCIA EMOCIONAL En EL AJUSTE ESCOLAR AUTOPERCIBIDO}

\section{Predictive ability of self-concept and emotional intelligence in perceived school adjustment}

IRATXE ANTONIO-AGIRRE, LOREA AZPIAZU, IGOR ESNAOLAY MARTA SARASA

Universidad del País Vasco/Euskal Herriko Unibertsitatea (UPVIEHU)

D01: 10.13042/Bordon.2015.67401

Fecha de recepción: 09/04/20150 - Fecha de aceptación: 01/06/2015

Autor de contacto / Corresponding Author: Igor Esnaola Echániz. E-mail: igor.esnaola@ehu.eus

Fecha de publicación online: 23/06/2015

INTRODUCCIÓN. La diversidad de capacidades y exigencias que acoge la escuela es un reto vigente. Como uno de los principales contextos implicados en el desarrollo psicosocial de los jóvenes, la escuela debe favorecer competencias sociopersonales como el autoconcepto y la inteligencia emocional para facilitar la adaptación de los jóvenes a un entorno educativo cambiante. El objetivo de este estudio es analizar la capacidad predictiva del autoconcepto multidimensional (físico, social, personal, académico y general) y la inteligencia emocional (atención, claridad y reparación) en el ajuste escolar percibido. MÉTODO. La muestra está compuesta por 1.543 adolescentes, 728 chicos $(47,18 \%)$ y 815 chicas $(52,81 \%)$ entre los 12 y los 18 años $\left(M_{\text {edad }}=14.24\right.$ y DT $\left.=1.63\right)$ de centros públicos y privados de Educación Secundaria de la Comunidad Autónoma Vasca. La información recogida se basa en tres autoinformes que miden las percepciones que tienen los participantes de su autoconcepto, sus capacidades emocionales y su ajuste escolar. Los análisis estadísticos se realizan a través del programa estadístico SPSS 21 para Windows. RESULTADOS. Los resultados indican que en la muestra masculina las escalas de reparación emocional, autoconcepto académico, autoconcepto social y autoconcepto general predicen significativamente el ajuste escolar de los adolescentes. En la muestra femenina, únicamente las escalas académica, personal y general del autoconcepto predicen significativamente el ajuste escolar. DISCUSIÓN. Estos resultados señalan la mayor importancia del autoconcepto en el ajuste escolar adolescente en comparación a la inteligencia emocional percibida.

Palabras clave: Ajuste escolar, Autoconcepto, Inteligencia emocional, Análisis de regresión múltiple. 


\section{Introducción}

La heterogeneidad de capacidades y necesidades que acogen las aulas constituye el mayor reto al que se enfrenta la escuela. Así lo señala la UNESCO (2009) en su $48^{\mathrm{a}}$ Conferencia Internacional de Educación que, tras varias décadas de iniciativas destinadas a favorecer una educación inclusiva y de calidad, pone de manifiesto la vigencia de este desafío planteado en las últimas décadas. De este modo, la escuela, como uno de los principales contextos implicados en el desarrollo psicosocial de los jóvenes, debe contribuir en el desarrollo máximo de sus capacidades y en la adquisición de recursos, oportunidades y experiencias significativas que favorezcan su adaptación a los requerimientos de un contexto escolar en continuo cambio.

El modelo centrado en el déficit, predominante en el siglo XX en las ciencias sociales y de la salud, ha dificultado una interpretación positiva del desarrollo de competencias sociopersonales en la escuela, como el autoconcepto y la inteligencia emocional (IE), a modo de posibles factores promotores de la adaptación al entorno y del bienestar personal. Por tanto, dejar atrás el paradigma educativo tradicional ha supuesto tomar en consideración otros marcos de referencia, como la psicología positiva (Seligman y Csikszentmihalyi, 2000), para comprender el ajuste escolar como resultado de la interacción conjunta escuela-familia-individuo.

Bajo este planteamiento, cabe entender el ajuste escolar como el grado de éxito en los intentos de los niños, niñas y adolescentes por adaptarse a las tareas y a las demandas cambiantes del entorno escolar, dadas las capacidades contextuales y los atributos personales de los que disponen (Ladd, 1989, 1990). Ladd, Kochenderfer-Ladd y Rydell (2011) sugieren una aproximación holística al ajuste escolar en la destacan como indicadores del éxito sociopersonal del alumnado para adaptarse a la escuela, las percepciones y valoraciones sobre la escuela, el bienestar psicológico en la escuela, la implicación escolar y el rendimiento académico.

A pesar de que la perspectiva multidimensional del ajuste escolar ha encontrado aceptación general entre la comunidad científica, no existe un consenso generalizado sobre los factores que lo componen (Martínez-Ferrer, 2008). Resultado de esta discrepancia conceptual son los numerosos y variados instrumentos empleados en la evaluación de este constructo. Precisamente, el rendimiento académico, medido mediante las calificaciones obtenidas por los estudiantes, ha sido una de las medidas del ajuste escolar por excelencia que más ampliamente ha sido analizada (Adell, 2002). Otras medidas habitualmente empleadas en la evaluación del ajuste escolar son el clima escolar (Bradshow, Waasdorp, Debnan y Johnson, 2014); la satisfacción escolar (Huebner, Hills, Siddal y Gilman, 2014); el apoyo social de padres, pares y profesores y la calidad de estas relaciones (Rodríguez-Fernández, Droguet y Revuelta, 2012; Roorda, Koomen, Spilt y Oort, 2011); y problemas internalizantes como el estrés, la depresión o la soledad (Gutiérrez y Gonçalves, 2013; Heras y Navarro, 2012) o problemas externalizantes, entre los que destacan el abandono escolar, el bullying o el consumo de sustancias psicoactivas (Martínez-Ferrer, Povedano, Amador y Moreno-Ruiz, 2012; Sánchez-Sosa, Villarreal, Ávila, Vera y Musitu, 2014).

\section{La variabilidad del ajuste escolar}

Las diferencias en el ajuste escolar en relación al género y a la edad han sido objeto habitual de estudio. Estudios previos señalan que las mujeres acostumbran a estar más integradas socialmente en la escuela y que obtienen calificaciones más altas que los hombres (Cava, Musitu, Buelga, y Murgui, 2010; Hernando, Oliva, y Pertegal, 2013). Sin embargo, otros trabajos cuestionan estas afirmaciones; Wang, Willett y Eccles (2011) ponen de manifiesto la 
ausencia sustancial de diferencias entre géneros en cuanto a su implicación escolar. Asimismo, Linver, Davis-Kean y Eccles (2002) señalan que investigaciones previas evidencian que los resultados dispares en el rendimiento académico entre chicos y chicas no solo han disminuido en las últimas décadas, sino que estas diferencias en sus resultados académicos serían solo atribuibles a subáreas específicas en las habilidades espaciales y verbales.

Respecto a la relación entre el ajuste escolar y la edad, las investigaciones precedentes han fundamentado la creencia preponderante que defiende la existencia de un patrón continuo de descenso en la implicación de los jóvenes a lo largo de los años que pasan en la escuela (Fredricks, Blumenfeld y Paris, 2004; Li y Lerner, 2011). En este sentido, los estudiantes de menor edad mostrarían actitudes más positivas hacia la escuela y sus expectativas de futuro, disminuyendo su implicación con el contexto escolar a medida que pasan de curso (Fredricks et al., 2004); del mismo modo, su rendimiento académico también descendería, aunque este declive sería más acusado entre aquellos estudiantes que además experimentasen una disminución en su participación en la escuela y en su aprendizaje autorregulado (Wang y Eccles, 2011). En todo caso, la investigación previa muestra que los resultados obtenidos hasta el momento son dispares en cuanto a la relación del ajuste escolar con el género, así como con la edad.

\section{El autoconcepto y el ajuste escolar}

El autoconcepto es uno de los factores individuales de mayor tradición en el estudio del ajuste escolar. Así, la imagen que tiene cada persona de sí misma es un constructo teórico estrechamente relacionado con el ajuste psicosocial en la adolescencia (Fuentes, García, Gracia y Lila, 2011), así como un factor personal determinante en el rendimiento académico (Adell, 2002).
Respecto a este constructo, también es necesario destacar que no se ha logrado una coincidencia de opinión en cuanto al número e identidad de los dominios que conforman el autonconcepto, ni tampoco respecto a las dimensiones de cada dominio (Goñi y Fernández, 2008). Una de las conceptualizaciones mayormente aceptada es el modelo jerárquico y multidimensional del autoconcepto de Shavelson, Hubner y Stanton (1976) que contempla un autoconcepto general formado por el autoconcepto académico y el autoconcepto no académico, que a su vez agruparía dominios como el autoconcepto social, emocional o físico.

En cuanto a los antecedentes, un amplio conjunto de estudios sostienen que el autoconcepto académico constituye la dimensión que mayor relación manifiesta con el éxito escolar, mientras que habría "una escasa o nula correlación entre el rendimiento académico y las facetas no académicas del autoconcepto" (Goñi y Fernández, 2008: 44). Pese a ello, algunas investigaciones informan de asociaciones significativas entre el autoconcepto social y el rendimiento académico, las conductas prosociales y el apoyo que los estudiantes perciben del grupo de pares (Amador y Mateos, 2000; Valdés, Sánchez-Escobedo, y Carlos-Martínez, 2012).

\section{La inteligencia emocional y el ajuste escolar}

Otra de las características individuales en auge actualmente en la investigación psicoeducativa es la inteligencia emocional, dado que el conocimiento que tiene cada persona de sus habilidades emocionales parece tener un papel relevante en su desarrollo sociopersonal y escolar (Ferragut y Fierro, 2012; Palomera, Salguero y Ruiz-Aranda, 2012).

Este constructo presenta dos aproximaciones teóricas principales: por una parte, el modelo de habilidades que conceptualiza la IE como procesos mentales que intervienen en la percepción, 
uso, comprensión y regulación de los estados emocionales para favorecer la resolución de problemas y la regulación del comportamiento (Mayer y Salovey, 1997; Salovey y Mayer, 1990); por otro lado, los modelos mixtos de la inteligencia emocional que incluyen habilidades y competencias emocionales percibidas y rasgos de la personalidad.

El cuestionario Trait Meta-Mood Scale (TMMS; Salovey, Mayer, Goldman, Turvey y Palfai, 1995), empleado en este estudio, es una de las medidas de inteligencia emocional percibida más utilizada (Pérez-González, Petrides y Furnham, 2007). A pesar de ser el TMMS una escala que operativiza la formulación original de la IE de Salovey y Mayer (1990), este instrumento se enmarca en los modelos mixtos de la IE dado que mide las creencias que tienen los individuos acerca de la atención que le dedican a sus sentimientos (atención emocional); la claridad de su experiencia emocional (claridad emocional) y el cese de los estados de ánimo negativos o la prolongación de los positivos (reparación emocional). Es más, Salguero, Fernández-Berrocal, Balluerka y Aritzeta (2010) señalan que estas dimensiones del TMMS podrían ayudar a explicar las diferencias individuales en el ajuste psicosocial adolescente. No obstante, son escasos los trabajos que proporcionan evidencias sobre la aportación diferencial de la atención, claridad y reparación emocional sobre el ajuste escolar. Esto podría, en parte, deberse a la relación inconsistente e incluso cuestionable que muestra la literatura previa entre la inteligencia emocional y el rendimiento académico (Jiménez-Morales y LópezZafra, 2013; Saklofske, Austin, Mastoras, Beaton, y Osborne, 2012); la evidencia teórica y empírica acumulada hasta el momento parece indicar que la inteligencia emocional medida mediante autoinformes presenta correlaciones bajas a moderadas con el desempeño académico (Perera y DiGiacomo, 2013).

En todo caso, la investigación previa muestra que los resultados obtenidos hasta el momento se presentan heterogéneos en cuanto a la relación del ajuste escolar con el autoconcepto y la inteligencia emocional. En este sentido, una mayor investigación podría ayudar a clarificar la aportación de estas variables personales sobre las distintas facetas del ajuste escolar a lo largo de la educación secundaria; y, asimismo, a tomar en consideración factores como el género o la edad en la adaptación de los adolescentes al contexto escolar.

Con todo esto, en relación a los antecedentes aquí expuestos, este trabajo tiene como objetivo general estudiar las relaciones entre el autoconcepto, la inteligencia emocional y el ajuste escolar. Los objetivos específicos de este estudio son los siguientes: (1) analizar las diferencias en el ajuste escolar en función del género; (2) examinar las diferencias en el ajuste escolar en relación a las etapas temprana (de 11 a 14 años) y media (de 15 a 18 años) de la adolescencia (Elliott y Feldman, 1990); y (3) analizar la capacidad predictiva del autoconcepto y de la inteligencia emocional sobre el ajuste escolar. En torno a cada uno de estos objetivos específicos se formulan las tres siguientes hipótesis:

H1: Las chicas presentarán niveles más altos de ajuste escolar, en comparación con el grupo de los chicos.

H2: Los adolescentes tempranos mostrarán niveles más altos de ajuste escolar, en comparación con los participantes pertenecientes a la etapa media de la adolescencia.

H3: Tanto el autoconcepto como la inteligencia emocional presentarán capacidad predictiva sobre el ajuste escolar de los participantes.

\section{Método}

\section{Participantes}

La muestra inicial está compuesta por 1.819 participantes de centros públicos y privados 
desde $1^{\circ}$ de la Educación Secundaria Obligatoria (ESO) hasta el $2^{\circ}$ curso de Bachillerato. Se trata de una muestra por conveniencia donde, después de la imputación de los valores perdidos, se realiza el análisis de normalidad multivariada, identificándose los valores extremos y procediendo a la eliminación de los valores atípicos, un total de 276 sujetos. Finalmente, la muestra asciende a 1.543 alumnos, de entre los cuales $728(47,18 \%)$ son chicos y $815(52,81 \%)$ son chicas; en función de la edad, 879 (56,96\%) constituyen el grupo de 11-14 años y 664 (43,03\%) constituyen el grupo de 15-18 años.

\section{Instrumentos}

En esta investigación se administran los siguientes cuestionarios:

- El Cuestionario de Autoconcepto Dimensional (AUDIM; Fernández-Zabala, Goñi, Rodríguez-Fernández y Goñi, en prensa) es un instrumento de medida que se enmarca dentro del modelo de Shavelson et al. (1976), con la salvedad de que opta por utilizar el término autoconcepto personal en lugar de autoconcepto emocional, por resultar más comprensivo y englobar el dominio emocional del autoconcepto junto a la percepción que tiene cada persona respecto al logro de sus objetivos de vida, su honradez y su capacidad para tomar decisiones en función de su propio criterio (Goñi y Fernández, 2008). Por lo tanto, este autoinforme consta de 33 ítems agrupados en cinco dimensiones: (1) Autoconcepto físico $(\alpha=.75)$, percepción particular de la forma física propia y de las habilidades y cualidades para la práctica de actividad físico-deportiva, junto con la percepción de la apariencia física propia y el grado en que la persona se ve o se siente físicamente fuerte. Esta subescala está compuesta por 8 ítems, 2 para cada una de sus cuatro subdimensiones (habilidad, condición, atractivo y fuerza); (2) Autoconcepto social $(\alpha=.67)$, percepción propia de la competencia social a la hora de desenvolverse en las relaciones e interacción con otras personas, así como a la percepción de responsabilidad social. Esta subescala está compuesta por 7 ítems (3 de ellos para la subdimensión responsabilidad social y los 4 restantes para la subdimensión aceptación social); (3) Autoconcepto personal $(\alpha=.59)$, percepción de uno mismo como persona íntegra y en la que se puede confiar, independiente de los demás, aparte de incluir la autopercepción de los aspectos más impulsivos y reactivos propios (emociones). Presenta un total de 8 ítems, a razón de 2 por cada una de las cuatro subdimensiones que la componen (honradez, autonomía, emociones y autorrealización); (4) Autoconcepto académico $(\alpha=.87)$, la percepción que el sujeto tiene de sí mismo como estudiante y en su desempeño de aprendizaje. Esta subsescala está formada por 6 ítems; y (5) Autoconcepto general $(\alpha=.84)$. El formato de respuesta es de tipo Likert con cinco opciones de respuesta y la consistencia interna de la escala global es de $\alpha=.82$.

- La escala de inteligencia emocional percibida, denominada Trait Meta-Mood Scale-24 (TMMS-24), es una adaptación al castellano realizada por FernándezBerrocal et al. (1998) a partir de la desarrollada por Salovey et al. (1995). Se trata de un autoinforme de 24 ítems que agrupa tres escalas: (1) Atención a los sentimientos $(\alpha=.89)$, grado en el que las personas creen prestar atención a sus sentimientos; (2) Claridad en los sentimientos $(\alpha=.86)$, comprensión de los propios estados emocionales; es decir, la manera en que el sujeto cree percibir sus propias emociones; y, (3) Reparación emocional $(\alpha=.83)$, creencia del sujeto en su capacidad para interrumpir estados emocionales negativos y prolongar 
los positivos. Estas tres escalas son evaluadas cada una de ellas a través de 8 ítems, proporcionando una puntuación por cada una de ellas. Sin embargo, este instrumento no proporciona una puntuación global.

- La Escala Breve de Ajuste Escolar (EBAE10), de Moral, Sánchez-Sosa y VillarrealGonzález (2010), es un autoinforme multidimensional del ajuste escolar que consiste en 10 ítems con seis opciones de respuesta agrupados en tres indicadores del ajuste escolar: (1) Problemas de integración $(\alpha=.84)$, se refiere a la relación con los compañeros y docentes y consta de 5 ítems en sentido inverso; (2) Rendimiento académico $(\alpha=.78)$ consta de 3 ítems; y, (3) Expectativas académicas $(\alpha=.85)$, se refiere a las expectativas y metas académicas de los estudiantes, conceptualizados como los propósitos de los estudiantes en relación con las cosas que quieren en el ámbito educativo y por las cuales se comprometen a realizar las tareas académicas. Este índice de ajuste escolar consta de 2 ítems. Este instrumento proporciona una puntuación global para el ajuste escolar de manera global y para cada uno de sus indicadores.

\section{Procedimiento y análisis de datos}

Primeramente, se pide el permiso ético pertinente para la realización del estudio, obteniendo un informe favorable de la Comisión de Ética de la Investigación y la Docencia (CEID) de la UPV/EHU. Tras haber establecido contacto telefónico, se solicita la autorización y conformidad de los centros educativos para la participación en el estudio. Al tratarse de menores de edad, se requiere a los tutores legales el consentimiento escrito a través de un documento elaborado específicamente para este propósito. La recogida de datos se desarrolla con cada grupo en una sesión de 45 minutos y es supervisada en la práctica totalidad de los casos por un investigador formado para tal fin. Con el propósito de reducir el efecto de deseabilidad social y la obtención de respuestas no sinceras, se insiste en la voluntariedad de la participación en el estudio y se asegura a los encuestados el procesamiento totalmente anónimo de los datos obtenidos. Se aplica el procedimiento de ciego simple, para evitar que los participantes conozcan el fin de la investigación, con el fin de reducir la posible influencia que podría tener en el resultado el conocimiento que el propio sujeto tendría sobre el factor en estudio.

Los análisis estadísticos de esta investigación son realizados con el programa estadístico SPSS 21 para Windows. Tras realizar la prueba de Kolmogorov-Smirnov, se comprueba que los datos resultantes de las escalas analizadas no se distribuyen normalmente y que no cumplen las condiciones paramétricas necesarias, por lo que se utiliza la prueba Mann-Whitney para analizar las diferencias entre grupos. Posteriormente, se emplean análisis de regresiones lineales múltiples para analizar la capacidad predictiva de los distintos dominios del autoconcepto y de la inteligencia emocional en el ajuste escolar percibido.

\section{Resultados}

Primeramente se exponen los resultados de la prueba Kolmogorov-Smirnov para las diferentes escalas aplicadas en este estudio: autoconcepto académico $\left(Z_{\mathrm{Ks}(\mathrm{Normal})}=3.31 ; p=.000\right)$; autoconcepto social $\left(Z_{\mathrm{Ks} \text { (Normal })}=4.23 ; p=.000\right)$; autoconcepto físico $\left(Z_{\mathrm{KS}(\mathrm{Normal})}=2.60 ; p=.000\right)$; autoconcepto personal $\left(Z_{\mathrm{KS}(\mathrm{Normal})}=2.90\right.$; $p=.000)$; autoconcepto general $\left(Z_{\mathrm{KS}(\mathrm{Normal})}=3.98\right.$; $p=.000)$; atención emocional $\left(Z_{\mathrm{KS}(\mathrm{Normal})}=1.81\right.$; $p=.003)$; claridad emocional $\left(Z_{\mathrm{KS}(\mathrm{Normal})}=1.53\right.$; $p=.018) ;$ reparación emocional $\left(Z_{\mathrm{Ks}(\mathrm{Normal})}=\right.$ $2.09 ; p=.000)$; rendimiento académico $\left(Z_{\mathrm{KS} \text { (Normal) }}\right.$ $=3.90 ; p=.000) ;$ problemas de integración $\left(Z_{\mathrm{KS} \text { (Normal) }}=5.26 ; p=.000\right) ;$ expectativas académicas $\left(Z_{\mathrm{Ks}(\text { Normal })}=10.95 ; p=.000\right) ; \mathrm{y}$ ajuste 
escolar global $\left(Z_{\mathrm{KS}(\mathrm{Normal})}=3.04 ; p=.000\right)$. Los resultados indican que ninguna de las escalas analizadas sigue una distribución normal.

A continuación, se examinan las diferencias de género y de edad en las diferentes escalas del ajuste escolar (tablas 1 y 2 ).

En cuanto a los adolescentes de entre 11 y 14 años, los datos señalan diferencias significativas en función del género. Estos resultados indican que las chicas correspondientes al grupo de adolescentes más jóvenes presentan un mayor nivel de ajuste escolar global, mayores expectativas académicas y mayor rendimiento académico percibido que los chicos; además, los participantes masculinos presentan puntuaciones que indican un peor nivel de integración en la escuela, en comparación con el de las chicas.

En relación a los adolescentes de entre 15 y 18 años, los datos muestran diferencias significativas entre géneros en el ajuste escolar de los adolescentes correspondientes a la etapa media de la adolescencia, similares a las encontradas en el grupo de adolescentes tempranos.

Después de analizar las diferencias en función del género, se examinan las diferencias entre los dos grupos de edad. Teniendo en cuenta que en los resultados anteriores se hallan diferencias significativas en función del género, se analizan los datos de forma independiente para cada uno de ellos (tabla 2).

\section{Tabla 1. Resultados en función del género}

\begin{tabular}{|c|c|c|c|c|c|}
\hline & & & & & \\
\hline & & Chicos & Chicas & Chicos & Chicas \\
\hline Rendimiento & M & 12.03 & 12.56 & 10.84 & 11.85 \\
\hline académıco & $(D T)$ & $(3.20)$ & $(2.98)$ & $(2.98)$ & $(3.15)$ \\
\hline & $z$ & & & & \\
\hline & $p$ & & & & \\
\hline Problemas de & $M$ & 10.36 & 9.60 & 10.16 & 9.06 \\
\hline & $(D T)$ & (3.92) & $(3.72)$ & $(3.59)$ & (3.36) \\
\hline & $z$ & & & & \\
\hline & $p$ & & & & \\
\hline Expectativas & M & 10.26 & 10.84 & 10.30 & 10.84 \\
\hline & $(D T)$ & (2.19) & (1.79) & $(2.02)$ & (1.82) \\
\hline & $z$ & & & & \\
\hline & $p$ & & & & \\
\hline Ajuste escolar & $M$ & 49.19 & 50.33 & 47.96 & 49.85 \\
\hline global & $(D T)$ & $(5.16)$ & (4.94) & (4.99) & $(5.10)$ \\
\hline & $z$ & & & & \\
\hline & $p$ & & & & \\
\hline
\end{tabular}

Nota: ${ }^{*} p<.05,{ }^{* *} p<.01,{ }^{* * *} p<.001$. 
Tabla 2. Resultados en función de los grupos de edad

\begin{tabular}{|c|c|c|c|c|c|}
\hline & & \multicolumn{2}{|c|}{ Chicos } & \multicolumn{2}{|c|}{ Chicas } \\
\hline & & 11-14 años & 15-18 años & 11-14 años & 15-18 años \\
\hline \multirow{4}{*}{$\begin{array}{l}\text { Rendimiento } \\
\text { académico }\end{array}$} & M & 12.03 & 10.84 & 12.56 & 11.85 \\
\hline & $(D T)$ & $(3.20)$ & (2.98) & $(2.98)$ & (3.15) \\
\hline & $z$ & \multicolumn{2}{|c|}{-5.37} & \multicolumn{2}{|c|}{-3.00} \\
\hline & $p$ & \multicolumn{2}{|c|}{$.000^{* * *}$} & \multicolumn{2}{|c|}{$.003 * * *$} \\
\hline \multirow{4}{*}{$\begin{array}{l}\text { Problemas de } \\
\text { integración }\end{array}$} & M & 10.36 & 10.16 & 9.60 & 9.06 \\
\hline & $(D T)$ & $(3.92)$ & (3.59) & $(3.72)$ & $(3.36)$ \\
\hline & $z$ & \multicolumn{2}{|c|}{-.296} & \multicolumn{2}{|c|}{-1.828} \\
\hline & $p$ & \multicolumn{2}{|c|}{.767} & \multicolumn{2}{|c|}{.068} \\
\hline \multirow{4}{*}{$\begin{array}{l}\text { Expectativas } \\
\text { académicas }\end{array}$} & M & 10.26 & 10.30 & 10.84 & 10.84 \\
\hline & $(D T)$ & (2.19) & $(2.02)$ & $(1.79)$ & $(1.82)$ \\
\hline & $z$ & \multicolumn{2}{|c|}{-.042} & \multicolumn{2}{|c|}{-.350} \\
\hline & $p$ & \multicolumn{2}{|c|}{.967} & \multicolumn{2}{|c|}{.726} \\
\hline \multirow{4}{*}{$\begin{array}{l}\text { Ajuste escolar } \\
\text { global }\end{array}$} & M & 49.19 & 47.96 & 50.33 & 49.85 \\
\hline & $(D T)$ & $(5.16)$ & (4.99) & $(4.94)$ & (5.10) \\
\hline & $z$ & \multicolumn{2}{|c|}{-3.26} & \multicolumn{2}{|c|}{-1.09} \\
\hline & $p$ & \multicolumn{2}{|c|}{$.001^{* * *}$} & \multicolumn{2}{|c|}{.274} \\
\hline
\end{tabular}

Nota. ${ }^{*} p<.05 .{ }^{* *} p<.01 .{ }^{* * *} p<.001$

Los datos muestran diferencias significativas entre los dos grupos de adolescentes analizados. De este modo, entre los chicos, los adolescentes del grupo de menor edad presentan mayores niveles en las escalas rendimiento académico y ajuste escolar global que el grupo de adolescentes de entre 15 y 18 años; mientras que en las chicas, tan solo en las adolescentes de entre 11 y 14 años se observan niveles más altos en la escala rendimiento académico.

En definitiva, los datos informan de mayores puntuaciones en el rendimiento académico en la etapa temprana de la adolescencia de los chicos y las chicas. Por el contrario, los niveles más altos de ajuste escolar global se hallan tan solo entre los chicos, en los adolescentes de entre 11 y 14 años. Aunque las chicas pertenecientes al grupo de menor edad también presentan puntuaciones mayores de ajuste escolar global que las adolescentes más mayores, estas diferencias no se muestran significativas.

Para la estimación de la capacidad predictiva de los dominios del autoconcepto y de la inteligencia emocional sobre el ajuste escolar se realizan análisis de regresión múltiple. Al haberse hallado diferencias significativas en las medias en función del género, se realizan dos análisis de regresión, uno para los chicos 
y otro para las chicas (tabla 3), introduciendo en cada uno de ellos simultáneamente todas las variables independientes (el autoconcepto académico, el autoconcepto social, el autoconcepto físico, el autoconcepto personal, el autoconcepto general, la atención emocional, la claridad emocional y la reparación emocional) que pudieran explicar cada uno de los indicadores de ajuste escolar empleados (el rendimiento académico, los problemas de integración, las expectativas académicas y el ajuste escolar global).

En cuanto a la capacidad predictiva del autoconcepto sobre los indicadores de ajuste escolar, los resultados de los chicos muestran la asociación significativa del autoconcepto académico con el rendimiento académico, los problemas de integración, las expectativas académicas y el ajuste escolar global; así como la asociación significativa del autoconcepto social y el autoconcepto global con el rendimiento académico, los problemas de integración y el ajuste escolar global.

En lo referente a la capacidad predictiva del autoconcepto sobre las escalas que miden el ajuste escolar de las chicas, los resultados muestran la asociación significativa del autoconcepto académico con el rendimiento académico, los problemas de integración, las expectativas académicas y el ajuste escolar global; otra asociación significativa es la del autoconcepto social con los problemas de integración; también se asociaría significativamente el autoconcepto personal con el rendimiento académico, las expectativas académicas y el ajuste escolar global; finalmente, se observa la asociación significativa del autoconcepto general con los problemas de integración y el ajuste escolar global.
Asimismo, los datos indican que, tanto en el grupo de los chicos como en el de las chicas, el autoconcepto académico es la variable independiente que más contribuye a explicar el rendimiento académico, las expectativas académicas y el ajuste escolar global, mientras que el autoconcepto general sería la variable independiente más relevante en la predicción de los problemas de integración.

En relación a la capacidad predictiva de la inteligencia emocional sobre los indicadores de ajuste escolar, los resultados de los chicos muestran la asociación significativa de la atención emocional con el rendimiento académico y los problemas de integración; también es necesario señalar la asociación significativa de la claridad emocional con el rendimiento académico; y, por último, se observa la asociación significativa de la reparación emocional con el rendimiento académico y el ajuste escolar global. En este caso, la reparación emocional sería la variable independiente más relevante en la predicción del rendimiento académico y el ajuste escolar global.

Por último, cabe señalar que los datos expuestos en la tabla 4 ponen de manifiesto una relación lineal significativa entre el conjunto de variables independientes y cada uno de los indicadores de ajuste escolar estudiados, por lo que resulta adecuado el empleo de procedimientos de regresión múltiple.

Por otra parte, estos resultados indican que los dominios del autoconcepto y de la inteligencia emocional explicarían el 38,5\% y el 35,2\% de la varianza del rendimiento académico y del ajuste escolar global, respectivamente, en el grupo de los chicos. Estas proporciones son similares en el caso de las chicas, aunque solo los dominios del autoconcepto contribuirían de forma significativa a explicar estas variables criterio. 


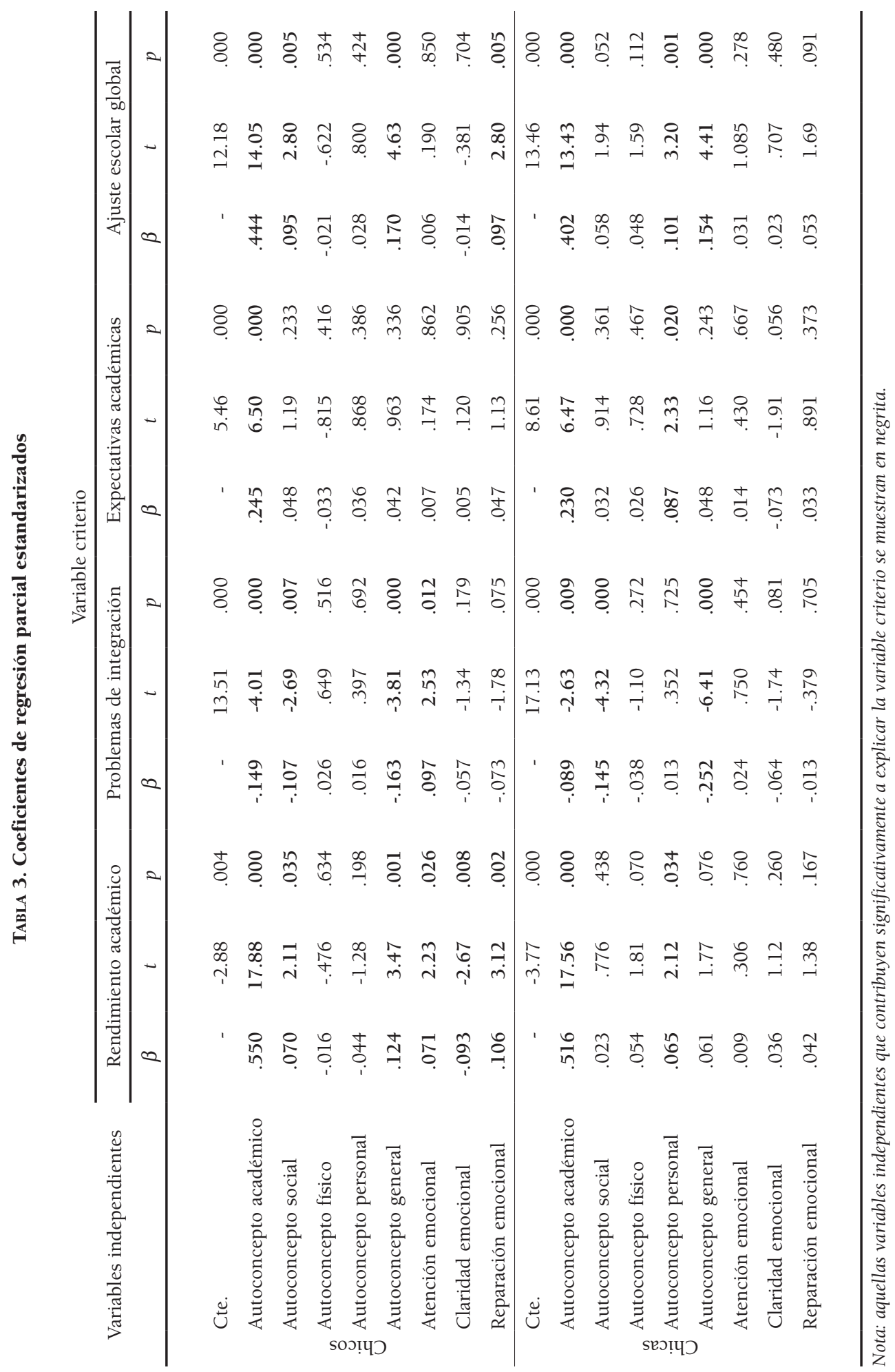


TABla 4. Capacidad predictiva de los modelos de regresión

\begin{tabular}{|c|c|c|c|c|c|}
\hline & & $F$ & $R$ & $R_{\text {corr }}^{2}$ & $S_{e}$ \\
\hline \multirow{4}{*}{$\begin{array}{l}\tilde{0} \\
\stackrel{0}{0} \\
\text { U. }\end{array}$} & Rendimiento académico & 60.91 & .636 & .385 & 2.46 \\
\hline & Problemas de integración & 12.57 & .350 & .109 & 3.57 \\
\hline & Expectativas académicas & 9.58 & .310 & .083 & 2.03 \\
\hline & Ajuste escolar global & 53.21 & .610 & .352 & 4.08 \\
\hline \multirow{4}{*}{$\stackrel{\mathscr{J}}{\stackrel{U}{U}}$} & Rendimiento académico & 67.02 & .632 & .385 & 2.39 \\
\hline & Problemas de integración & 23.30 & .433 & .170 & 3.24 \\
\hline & Expectativas académicas & 11.01 & .314 & .086 & 1.72 \\
\hline & Ajuste escolar global & 66.16 & .630 & .352 & 3.91 \\
\hline
\end{tabular}

Nota. El valor del nivel crítico asociado al estadístico $F$ es menor de $p<.001$ en todos los modelos analizados; $R_{\text {corr }}^{2}$ Coeficiente de determinación corregido; $S_{e}$ : Error típico de la estimación.

\section{Discusión}

El objetivo general del estudio es analizar las relaciones entre el autoconcepto multidimensional (físico, social, personal, académico y general), la inteligencia emocional (atención, claridad y reparación) y el ajuste escolar en la adolescencia.

En cuanto al primer objetivo específico, hay que señalar que los resultados encontrados en el estudio sobre las diferencias en función del género en el ajuste escolar confirman la primera hipótesis, dado que los mayores niveles de ajuste escolar de las chicas coincide con algunos estudios previos (Cava et al., 2010; Hernando et al., 2013).

En lo que respecta al segundo objetivo, tras analizar las diferencias de edad entre la adolescencia temprana y la adolescencia media, los resultados también corroboran la segunda hipótesis planteada. Los resultados evidencian que los más jóvenes en ambos géneros perciben niveles más altos de rendimiento académico; y en la muestra masculina también mayor grado de ajuste escolar global, coincidiendo con estudios previos (Li y Lerner, 2011; Wang y Eccles, 2011).

Por otro lado, respecto al tercer objetivo, el análisis realizado para examinar la capacidad predictiva del autoconcepto y la inteligencia emocional sobre el ajuste escolar confirma nuestra tercera hipótesis. En cualquier caso, es necesario matizar esta afirmación, ya que no todas las dimensiones del autoconcepto o la inteligencia emocional contribuyen en la predicción de los índices de ajuste escolar empleados en este estudio. En primer lugar, es necesario señalar que la dimensión académica y la general del autoconcepto presentan una capacidad significativa para predecir las puntuaciones en la mayoría de las escalas del ajuste escolar en ambos géneros, coincidiendo estos resultados con los estudios que señalan que dentro del modelo jerárquico y multidimensional del autoconcepto, la dimensión académica es la que mayor relación manifiesta con el éxito escolar (Goñi y Fernández, 2008). Estos autores señalan que las dimensiones no académicas obtienen relaciones escasas o nulas (Goñi y Fernández, 2008), aunque en nuestro caso la dimensión emocional predice significativamente tres de las 
cuatro escalas del ajuste escolar en la muestra masculina, mientras el autoconcepto personal lo hace en la muestra femenina. Asimismo, los datos aportan evidencia de la capacidad predictiva de la atención emocional, claridad emocional y reparación emocional sobre el rendimiento académico percibido de los adolescentes. No obstante, tan solo la percepción que tienen los jóvenes sobre su capacidad para regular sus emociones parece predecir significativamente su ajuste escolar global. Por último, resulta necesario señalar la aportación diferencial del autoconcepto y la inteligencia emocional al ajuste escolar percibido, dado que estos resultados recalcan la mayor aportación del autoconcepto a la predicción del ajuste escolar adolescente en comparación a la inteligencia emocional.

Este estudio tiene ciertas limitaciones, ya que futuros estudios podrían analizar la relación y capacidad predictiva de las dimensiones específicas del autoconcepto y la inteligencia emocional en el ajuste escolar a través de modelos de ecuaciones estructurales, así como realizar análisis multigrupo en función de la edad (adolescencia temprana, media y tardía), el género, la clase social, etc. Por otro lado, sería interesante que futuros estudios puedan inferir relaciones de "causalidad" entre otros constructos individuales (resiliencia, satisfacción con la vida, etc.) que ayuden a entender la influencia de dichas variables en el ajuste escolar en la adolescencia.

Asimismo, otra de las limitaciones del estudio deriva de haber evaluado el rendimiento académico percibido y no el real. Se sugiere emplear en futuras investigaciones criterios externos, como las evaluaciones realizadas por los profesores.

Finalmente, dado que los datos no siguen una distribución normal, el tener que utilizar pruebas no paramétricas podría considerarse otra limitación, ya que estas podrían restar solidez a los resultados.

A pesar de dichas limitaciones, este estudio aporta información sobre la relación del autoconcepto multidimensional y la inteligencia emocional con el ajuste escolar en la adolescencia. Estos resultados pueden ofrecer apoyo empírico para que se desarrollen programas de intervención que persigan mejorar el autoconcepto y la inteligencia emocional con el objetivo de que dicha mejora influya en el ajuste escolar.

\section{Nota}

${ }^{1}$ Esta investigación forma parte del trabajo realizado dentro del Grupo Consolidado de Investigación del Sistema Universitario Vasco IT701-13 y de los resultados del proyecto de investigación EHUA13/26 de la Universidad del País Vasco.

\section{Referencias bibliográficas}

Adell, M. A. (2002). Estrategias para mejorar el rendimiento académico de los adolescentes. Madrid: Pirámide.

Amador, L., y Mateos, F. (2000). La dimensión social del autoconcepto en el adulto y su relación con el rendimiento académico. Enseñanza: Anuario Interuniversitario de Didáctica, 17-18, 99-114.

Bradshaw, C. P., Waasdorp, T. E., Debnam, K. J., y Johnson, S. L. (2014). Measuring school climate in high schools: A focus on safety, engagement, and the environment. Journal of School Health, 84 (9), 593-604. 
Capacidad predictiva del autoconcepto y la inteligencia emocional en el ajuste escolar autopercibido

Cava, M. J., Musitu, G., Buelga, S., y Murgui, S. (2010). The relationships of family and classroom environments with peer relational victimization: An analysis of their gender differences. The Spanish Journal of Psychology, 13 (1), 156-165. doi: 10.1017/S1138741600003747

Elliott, G. R., y Feldman, S. S. (1990). Capturing the adolescent experience. En S. S. Feldman y G. R. Elliott (eds.), At the threshold: The developing adolescent (pp. 1-53). Cambridge, MA: Harvard University Press.

Fernández-Berrocal, P., Alcaide, R., Domínguez, E., Fernández-MacNally, C., Ramos, N., y Ravira, M. (1998). Adaptación al castellano de la escala rasgo de metaconocimiento sobre estados emocionales de Salovey et al.: Datos preliminares. En Libro de actas del V Congreso de Evaluación Psicológica (vol. 1, pp. 83-84).

Fernández-Zabala, A., Goñi, E., Rodríguez-Fernández, A., y Goñi, A. (en prensa). Un nuevo cuestionario en castellano con escalas de las dimensiones del autoconcepto. Revista Mexicana de Psicología, 32 (2).

Ferragut, M., y Fierro, A. (2012). Inteligencia emocional, bienestar personal y rendimiento académico en preadolescentes. Revista Latinoamericana de Psicología, 44 (3), 95-104.

Fredricks. J. A., Blumenfeld, P. C., y Paris, A. H. (2004). School engagement: Potential of the concept, state of the evidence. Review of Educational Research, 74 (1), 59-109.

Fuentes, M. C., García, J. F., Gracia, E., y Lila, M. (2011). Autoconcepto y ajuste psicosocial en la adolescencia. Psicothema, 23 (1), 7-12.

Goñi, E., y Fernández, A. (2008). El autoconcepto. En A. Goñi (coord.), El autoconcepto físico: Psicología y Educación (pp. 23-58). Madrid: Pirámide.

Gutiérrez, M., y Gonçalves, T. O. (2013). Activos para el desarrollo, ajuste escolar y bienestar subjetivo de los adolescentes. International Journal of Psychology and Psychological Therapy, 13 (3), 339-355.

Heras, J., y Navarro, R. (2012). Ajuste escolar, soledad y conducta agresiva entre estudiantes de educación secundaria. Revista Qurriculum, 25, 105-124.

Hernando, A., Oliva, A., y Pertegal, M. A. (2013). Diferencias de género en los estilos de vida de los adolescentes. Psychological Intervention, 22, 15-23. doi: 10.5093/in2013a3

Huebner, E. S., Hills, K. J., Siddall, J., y Gilman, R. (2014). Life satisfaction and schooling. En M. J. Furlong, R. Gilman y E. S. Huebner (eds.), Handbook of positive psychology in the schools ( $2^{\mathrm{a}} \mathrm{ed}$., pp. 192-208). Nueva York: Routledge.

Jiménez-Morales, M. I., y López-Zafra, E. (2013). Impacto de la inteligencia emocional percibida, actitudes sociales y expectativas del profesor en el rendimiento académico. Electronic Journal of Research in Educational Psychology, 11 (1), 75-98. Recuperado de http://www.investigacion-psicopedagogica.org/revista/new/ContadorArticulo.php?771

Ladd, G. W. (1989). Children's social competence and social supports: Precursors of early school adjustment? En B. J. Schneider, G. Attili, J. Nadel y R. Weissberg (eds.), Social competence in developmental perspective (pp. 277-291). Amsterdam: Kluwer.

Ladd, G. W. (1990). Having friends, keeping friends, making friends, and being liked by peers in the classroom: Predictors of children's early school adjustment? Child Development, 61 (4), 1081-1100.

Ladd, G. W., Kochenderfer-Ladd, B., y Rydell, A. M. (2011). Children's interpersonal skills and school-based relationships. En P. K. Smith y C. H. Hart (eds.), The Wiley-Blackwell handbook of childhood social development (pp. 181-206). Malden, MA: Wiley-Blackwell. doi: $10.1002 / 9781444390933$

Li, Y., y Lerner, R. M. (2011). Trajectories of school engagement during adolescence: Implications for grades, depression, delinquency, and substance use. Developmental Psychology, 47 (1), 233247. doi: 10.1037/a0021307 
Linver, M. R., Davis-Kean, P., y Eccles, J. E. (abril, 2002). Influences of gender on academic achievement. Trabajo presentado en la Biennial Meetings of the Society for Research on Adolescence, Nueva Orleans, LA.

Martínez-Ferrer, B. (2008). Ajuste escolar, rechazo y violencia en adolescentes (tesis doctoral). Valencia: Universidad de Valencia.

Martínez-Ferrer, B., Povedano, A., Amador, L. V., y Moreno-Ruiz, D. (2012). Clima escolar, satisfacción con la vida y victimización en la escuela: un análisis del efecto moderador del género. Anales de Psicología, 28 (3), 875-882.

Mayer, J. D., y Salovey, P. (1997). What is emotional intelligence? En P. Salovey y D. Sluyter (eds.), Emotional development and emotional intelligence: Implications for educators (pp. 3-31). Nueva York: Basic Books.

Moral, J. C., Sánchez-Sosa, J. C., y Villarreal-González, M. E. (2010). Desarrollo de una Escala Breve de Ajuste Escolar. Revista Electrónica de Metodología Aplicada, 15 (1), 1-11. Recuperado de http://www.unioviedo.es/reunido/index.php/Rema/article/view/9790/9529

Palomera, R., Salguero, J. M., y Ruiz-Aranda, D. (2012). La percepción emocional como predictor estable del ajuste psicosocial en la adolescencia. Psicología Conductual, 20 (1), 43-58.

Perera, H. N., y DiGiacomo, M. (2013). The relationship of trait emotional intelligence with academic performance: A meta-analytic review. Learning and Individual Differences, 28, 20-33.

Pérez-González, J. C., Petrides, K. V., y Furnham, A. (2007). La medida de la inteligencia emocional rasgo. En J. M. Mestre y P. Fernández-Berrocal (coords.), Manual de inteligencia emocional (pp. 81-97). Madrid: Pirámide.

Rodríguez-Fernández, A., Droguett, L., y Revuelta, L. (2012). School and personal adjustment in adolescence: The role of academic self-concept and perceived social support. Revista de Psicodidáctica, 17 (2), 397-414.

Roorda, D. L., Koomen, H. M., Spilt, J. L., y Oort, F. J. (2011). The influence of affective teacherstudent relationships on students' school engagement and achievement a meta-analytic approach. Review of Educational Research, 81 (4), 493-529.

Sánchez-Sosa, J. C., Villarreal, M. E., Ávila, M. E., Vera, A., y Musitu, G. (2014). Contextos de socialización y consumo de drogas ilegales en adolescentes escolarizados. Psicosocial Intervention, 23 (1), 69-78. doi: 10.5093/in2014a7

Saklofske, D. H., Austin, E. J., Mastoras, S. M., Beaton, L., y Osborne, S. E. (2012). Relationships of personality, affect, emotional intelligence and coping with student stress and academic success: Different patterns of association for stress and success. Learning and Individual Differences, 22, 251-257. doi: 10.1016/j.lindif.2011.02.010

Salguero, J. M., Fernández-Berrocal, P., Balluerka, N., y Aritzeta, A. (2010). Measuring perceived emotional intelligence in the adolescent population: Psychometric properties of the Trait Meta-Mood Scale. Social Behavior and Personality, 38 (9), 1197-1210. doi: 10.2224/sbp.2010.38.9.1197

Salovey, P., y Mayer, J. D. (1990). Emotional intelligence. Imagination, Cognition, and Personality, 9 , 185-211.

Salovey, P., Mayer, J. D., Goldman, S. L., Turvey, C., y Palfai, T. P. (1995). Emotional attention, clarity, and repair: Exploring emotional intelligence using the Trait Meta-Mood Scale. En J. W. Pennebaker (ed.), Emotion, disclosure, and health (pp. 125-154). Washington: American Psychological Association.

Seligman, M., y Csikszentmihalyi, M. (2000). Positive psychology: An introduction. American Psychologist, 55 (1), 5-14. doi: 10.1037/0003-066X.55.1.5

Shavelson, R. J., Hubner, J. J., y Stanton, J. C. (1976). Self-concept: Validation of construct interpretation. Review of Educational Research, 46, 407-441. 
Capacidad predictiva del autoconcepto y la inteligencia emocional en el ajuste escolar autopercibido

UNESCO (2009). Directrices sobre politicas de inclusión en la educación. París: UNESCO.

Valdés, A. A., Sánchez-Escobedo, P. A., y Carlos-Martínez, E. A. (2012). Autoconcepto social y ajuste escolar de estudiantes de educación media con conductas de hostigamiento en la escuela. Educación y Ciencia, Cuarta Época, 2 (5), 85-96.

Wang, M. T., y Eccles, J. S. (2011). Adolescent behavioral, emotional, and cognitive engagement trajectories in school and their relations to educational success. Journal of Research on Adolescence, 22 (1), 31-39. doi: 10.1111/j.1532-7795.2011.00753.x

Wang, M. T., Willett, J. B., y Eccles, J. S. (2011). The assessment of school engagement: Examining dimensionality and measurement invariance by gender and race/ethnicity. Journal of School Psychology, 49 (4), 465-480. doi: 10.1016/j.jsp.2011.04.001

Abstract

Predictive ability of self-concept and emotional intelligence in perceived school adjustment

INTRODUCTION. The diversity of skills and demands that school hosts for pupils is an ongoing challenge. As one of the main contexts implicated in the psychosocial development of young people, school should boost sociopersonal skills such as self-concept and emotional intelligence to facilitate the adaptation of youth to a changeable educational environment. The aim of this study is to analyze the predictive ability of multidimensional self-concept (physical, social, personal, academic, and general) and perceived emotional intelligence (attention, clarity, and repair) in school adjustment. METHOD. The sample is composed of 1543 adolescents, 728 boys $(47.18 \%)$ and 815 girls $(52.81 \%)$ between 12 and 18 years of age $\left(M_{\text {age }}=14.24\right.$ and $\left.S D=1.63\right)$ from public and private Secondary Education schools of the Basque Autonomous Community. The information collected is based on three self-reports that measure perceptions of participants' own self-concept, emotional skills, and school adjustment. The statistical analyses are performed through SPSS 21 for Windows. RESULTS. The results indicate that in the male sample emotional repair, academic self-concept, social self-concept, and general self-concept scales significantly predict school adjustment of the adolescents. In the female sample, only the academic, personal, and general self-concept scales significantly predict school adjustment. DISCUSSION. These results point out the greater importance of self-concept in adolescent school adjustment compared to perceived emotional intelligence.

Key words: School adjustment, Self-concept, Emotional intelligence, Multiple regression analysis.

\section{Résumé}

Capacité prédictive du concept de soi et de l'intelligence émotionnelle dans l'ajustement scolaire auto-perçu

INTRODUCTION. La diversité des capacités et des exigences existant à l'école représente un important défi dans l'actualité. Étant l'école une parmi des principaux contextes impliqués dans le développement psychosocial des jeunes, elle doit favoriser des compétences sociopersonnelles telles que le concept de soi et l'intelligence émotionnelle, afin de faciliter l'adaptation des jeunes au milieu éducatif changeant. L'objectif de cette étude est d'analyser la capacité prédictive 
du concept de soi d'après un point de vue multidimensionnelle (physique, sociale, personnelle, académique et générale), ainsi que d'analyser le rôle de l'intelligence émotionnelle (attention, clarté et réparation émotionnelle) dans l'ajustement scolaire perçu par les élèves. MÉTHODE. Léchantillon est composé de 1543 adolescents, 728 garçons (47.18\%) et 815 filles (52.81\%), entre 12 et 18 ans $\left(M_{\hat{a g e}}=14.24\right.$ et $\left.D T=1.63\right)$, provenant des écoles secondaires privées et publiques de la communauté autonome du Pays Basque. Linformation recueillie est basée sur trois auto-rapports qui mesurent les perceptions que les participants ont de soi, leurs capacités émotionnelles et leur ajustement scolaire. Les analyses statistiques sont réalisées à travers du logiciel SPSS 21 pour Windows. RÉSULTATS. Les résultats montrent que chez les garçons les échelles de réparation émotionnelle, le concept de soiacadémique, le concept de soi social, et concept de soi en général prédisent notablement l'ajustement scolaire. Chez les filles, ce sont les échelles académique, personnelle et générale du concept de soi qui prédisent l'ajustement scolaire d'une façon très significative. DISCUSSION. Ces résultats révèlent l'importance majeure du concept de soi dans l'ajustement scolaire par rapport à l'intelligence émotionnelle perçue.

Mots clés: Ajustement scolaire, Concept de soi, Intelligence émotionnelle, Analyse de la régression multiple.

\section{Perfil profesional de los autores}

\section{Iratxe Antonio-Agirre}

Investigadora predoctoral en el Departamento de Psicología Evolutiva y de la Educación de la Universidad del País Vasco. Le ha sido concedida una ayuda para la Formación de Personal Universitario (FPU13) del Ministerio de Educación, Cultura y Deporte de España para estudiar la influencia de factores personales y contextuales en el ajuste personal y escolar en la adolescencia.

Correo electrónico de contacto: iratxe.antonio@ehu.eus

\section{Lorea Azpiazu Izaguirre}

Investigadora predoctoral en el Departamento de Psicología Evolutiva y de la Educación de la Universidad del País Vasco. Le ha sido concedida una ayuda para la Formación de Personal Universitario de la Universidad del País Vasco para estudiar el ajuste escolar en la adolescencia.

Correo electrónico de contacto: lorea.azpiazu@ehu.eus

\section{Igor Esnaola Echániz (autor de contacto)}

Profesor agregado de la Universidad del País Vasco, ha impartido docencia en grado así como en el Máster de Psicodidáctica. Como miembro del grupo de investigación consolidado por el Gobierno Vasco Psikor, ha publicado una docena de artículos ISI en relación al autoconcepto, aunque actualmente se orienta más al análisis de las variables contextuales y personales influyentes en el ajuste personal-escolar.

Correo electrónico de contacto: igor.esnaola@ehu.eus

Dirección para la correspondencia: Facultad de Filosofía y Ciencias de la Educación, Departamento de Psicología Evolutiva y de la Educación. Universidad del País Vasco / Euskal Herriko Unibertsitatea. Avda. Tolosa, 70, C.P. 20018. 


\section{Marta Sarasa Maya}

Profesora del Departamento de Psicología Evolutiva y de la Educación de la Escuela Universitaria de Magisterio (UPV/EHU). Su labor se centra dentro del marco de la educación inclusiva y sobre todo en el ámbito de las necesidades educativas especiales y especialmente en la población con autismo y trastornos generalizados del desarrollo y sus familias.

Correo electrónico de contacto: marta.sarasamaia@ehu.eus 